\title{
LA CONFIANZA: APROXIMACIONES TEÓRICAS Y PROPUESTA SISTÉMICA PARA SU ABORDAJE EN LAS CIENCIAS SOCIALES
}

\author{
TRUST: THEORETICAL APPROXIMATIONS AND SYSTEMIC
} PROPOSED APPROACH FOR THE SOCIAL SCIENCES

César David Mariñez Sánchez ${ }^{1}$

"Without general trust that people have in each other society itself would disintegrate” (Simmel, 2011, p.191).

Resumen: El siguiente ensayo busca identificar a modo general las perspectivas teóricas sobre la cual se ha venido sosteniendo la producción de conocimiento sobre la confianza. Luego entraremos con una propuesta desde la Teoría de los Sistemas Sociales principalmente en Niklas Luhmann. Nos abriremos hacia un programa científico que tiene como propósito observar a la confianza como un concepto funcional, multidimensional, reflexivo y que opera en la complejidad.

Palabras claves: Confianza, Ciencias Sociales, Sistemas Sociales, Niklas Luhmann.

[1] Magíster en Análisis Sistémico Aplicado a la. Sociedad, Doctorando en Filosofía, Universidad de Chile, Santiago de Chile, e-mail: cdmarinez@u.uchile.cl 
ABSTRACT: The following essay seeks to identify in general the theoretical perspectives on which has been supporting the production of knowledge on trust. Then enter a proposal from the Theory of Social Systems mainly Niklas Luhmann. We will open to a scientific program that aims to observe trust as a functional concept, multidimensional, thoughtful and operating complexity.

Key-words: Trust, Social Sciences, Social Systems, Niklas Luhmann.

\section{Introducción:}

El fenómeno de la confianza ha venido siendo un concepto de gran interés para las ciencias sociales y humanas ya que cada vez más se observa que el compromiso o la cooperación en las relaciones sociales se hacen cada vez más improbable. Como bien lo planteó Peter Blau en su estudio sobre el intercambio y el poder en la vida social, "el problema inicial consiste en demostrar que uno mismo es una persona digna de confianza [...] implica el riesgo de rechazo de la apertura por medio de la negativa a corresponder y a entrar en una relación." (Blau, 1982, p. 82). Así, confiar en las otras personas ya no se concibe automáticamente como algo obvio (Luhmann, 1996) y como no se puede confiar en la gente, se confía en las instituciones como mediadores y generalizadores de la confianza (Offe, 1999). En otras palabras, la confianza no sólo se construye a partir de las relaciones interpersonales sino también sobre aquellas estructuras que permiten procesar las decepciones que cada vez más se hacen frecuentes en la vida social. Los roles que tenemos en la vida diaria o que vivenciamos en la cotidianeidad con el abogado, con el médico, con el profesor o con los sistemas sociales como la familia o el gobierno son necesarias para que puedan construirse expectativas de persistencia en la sociedad (Barber, 1983). Por lo tanto la confianza viene a ser un prerrequisito para el orden social en la medida 
en que los distintos sistemas sociales logran regular las decepciones que cada vez más se generalizan en el mundo actual.

Bajo este contexto, desde la sociología, la antropología, la psicología, las ciencias políticas o la misma filosofía práctica, se han venido desplegando una cantidad muy diversa de reflexiones o investigaciones sobre la confianza. Como lo menciona Barbara Mitzal (2001), "the research becomes more specialized, definitions more specific and now writers do not talk about trust in general, but rather about various categories of trust or about trust in different contexts, such as trust in organizations, political systems or in families". (Mitzal, 2001, p. 372). Estudios sobre la aplicación y conceptualización de la confianza en la administración (Choudhury, 2008), en la ciencia (Sztompka, 2007), en los espacios públicos democráticos (Mitzal, 2001), en las relaciones trans-organizacionales (Bachmann, 2001), en los espacios económicos (Murphy, 2006) o en las Relaciones Internacionales (Hoffmann, 2002) serían unos de los tantos estudios que se han venido realizando sobre dicho término en el campo de las Ciencias Sociales.

De acá se despliega una inquietud en el campo de las ciencias sociales para el abordaje del problema de la confianza. Si bien existe un acuerdo en la cual esta es necesaria o es un prerrequisito para mantener el orden social en sus diferentes contextos, cabría preguntarse si sus referencias teóricas y epistemológicas para la producción de conocimiento sobre la confianza social pueden tener rendimientos si vinculamos dichos planteamientos con la complejidad a la cual ha llegado la sociedad. Como veremos más adelante, en las ciencias sociales existe una tendencia a abordar el problema desde un enfoque ontológico o desde un enfoque psicologista de la sociedad, por lo que cabe preguntarse si tales estudios pueden tener rendimientos teniendo en cuenta lo dinámico que ha llegado ser el entorno en el que vivimos y los horizontes de posibilidad al cual nos enfrenamos en la vida diaria (Beriain, 2008).

Así, la siguiente investigación parte del supuesto que los estudios de corte atomicista o normativos dificultarían la posibilidad de 
observar el grado de la complejidad a la que ha llegado la estructura social, por lo que este trabajo intentará ofrecer una propuesta desde la Teoría de los Sistemas Sociales principalmente en Niklas Luhmann para el abordaje del fenómeno de la confianza social.

La investigación está dividida en tres partes: la primera parte busca describir el modo en que se ha venido tratado el tema de la confianza desde una visión "atonomicista" de la sociedad. Es decir, veremos la manera en que las investigaciones sólo se centran en las características individuales y en la cual sus análisis giran en torno a lo meramente cognitivo, conductual o interaccional. Como segunda parte de la investigación, describiremos el modo en que la confianza ha sido tratado en un plano más holista de la sociedad, es decir, "desde una perspectiva fundamentalmente ontológica con respecto a lo social" (Arnold y Rodríguez, 1990, p. 34) o como lo vino mencionando Horkheimer (1931) en sus primeros estudios en la Escuela Crítica, bajo una fundamentación de la totalidad social más allá del alcance de las ciencias empíricas. Bajo esta perspectiva, el análisis de la confianza no apunta a formularse la pregunta sobre cuáles son las condiciones que hacen posible la confianza a partir de las cualidades o de las características individuales o cognitivas de las personas, más bien se centran en el aspecto normativo como condición necesaria para poder producir confianza social. En la tercera parte nos centraremos en la confianza sistémica principalmente en la propuesta realizada por Niklas Luhmann. Creemos que su programa sobre la teoría de los sistemas sociales y su marco referencial puede tener mayores rendimientos para trabajar con el tema de la confianza ya que nos permite trabajar con mayor rigurosidad en la complejidad de lo social. Después de describir el modo en que la confianza ha venido siendo manipulada en un plano normativo o intersubjetivo y cognitivo o psicológico, nos abriremos hacia un programa científico que tiene como propósito observar a la confianza como un concepto funcional, multidimensional, reflexivo y que opera en la complejidad. 


\section{Acercamientos micro al estudio de la confianza:}

Podríamos catalogar de "atomicista" a aquella perspectiva en los cual los estudios "sólo enfatizan la acción individual" (Arnold; Rodríguez, 1990, p. 34). Sobre la pregunta de cuáles son las condiciones necesarias para se sostenga el orden social, el enfoque atomicista sugiere que aquello es posible por medio de las capacidades cognitivas de los individuos autónomos en sus relaciones interpersonales (Lewis; Weigert, 1985). La finalidad de todo comportamiento humano estaría entonces en utilizar sus capacidades cognitivas para relacionarse con los demás y para ellos la confianza mutua viene a ser la condición necesaria por la cual los hombres pueden dirigir sus acciones de una determinada manera para lograr sus fines e intereses.

Una de las corrientes filosóficas que ha contribuido a conceptualizar a la confianza desde este enfoque tiene que ver por ejemplo con la tradición empirista, quien en el terreno de la moral considera que el estudio sobre la naturaleza humana sería posible a través de un método experimental que permita identificar en el comportamiento humano los atributos o las cualidades por las cuales los hombres dirigen sus acciones de una determinada manera. Como bien lo menciona David Hume, el objetivo es buscar los hechos por las cuales se deriva en última instancia la aprobación o la censura de la conducta humana (Hume, 2003, p. 35). Siguiendo a David Lewis y Andrew Weigert (1985), la pregunta que se despliega desde esta corriente filosófica, es la de si los humanos tienen una inclinación natural para confiar o desconfiar en otro. Bien sea a través de la pasión o de la simpatía, el estado real de los asuntos humanos será el principal interés para preguntarse sobre cuáles son las condiciones de posibilidad de mantener la confianza mutua y la armonía social.

En Hobbes por ejemplo, el conocimiento también se funda en la experiencia. El autor parte de la idea de que los fenómenos son aprehendidos por los órganos sensoriales y por lo tanto el hombre desde un punto de vista mecanicista, es observado como alguien que se mueve por algo lo que lo atrae (pasiones) y se aleja de aquello 
que le desagrada (dolores). "No existen tendencias o esfuerzo que estén dirigidos hacia la realización de algo que no esté existiendo aún; ellos son meros movimientos, producidos por la acción de los cuerpos que ya existen, las acciones van por atracción o repulsión" (Collingwood, 2006, p.103). Bajo esta concepción mecanicista del hombre Hobbes va a sostener que:

"De la igualdad procede la desconfianza. De esta igualdad en cuanto a la capacidad se deriva la igualdad de esperanza respecto a la consecución de nuestros fines. Esta es la causa de que si dos hombres desean la misma cosa, y en modo alguno pueden disfrutarla ambos, se vuelven enemigos, y en el camino que conduce al fin (que es, principalmente, su propia conservación, y a veces su delectación tan sólo) tratan de aniquilarse o sojuzgarse uno a otro" (Hobbes, 1997, p.128).

A diferencia de Hume en la cual el deseo es lo que produce la armonía social, en Hobbes el deseo causa conflicto ya que es lo que a los hombres los atrae por naturaleza. Por esa razón, "la causa del miedo mutuo reside en parte en la igualdad natural de los hombres, en parte en la voluntad de hacerse daño mutuamente" (Hobbes, 2010, p.133). Para Hobbes, la solución que da a esta lucha de todos contra todos o al estado de naturaleza es a través de un contrato con el poder soberano ya que sólo así es posible mantener la paz y la armonía de intereses.

Volviendo al plano de las ciencias sociales, el estado real de los asuntos humanos seguirá siendo el principal interés para preguntarse sobre cuáles son las condiciones de posibilidad de mantener la confianza mutua en las relaciones interpersonales. Se le pondrá el foco en el modo en que se construyen las expectativas personales de los actores individuales en una relación interpersonal. Para ello, se busca predecir los comportamientos humanos a través de experimentos psicosociales o escalas psicométricas, para determinar el 
modo en que los hombres se comportan de una determinada manera. Para caracterizar esta perspectiva para el estudio de lo social tomaremos como ejemplo a Morton Deutsh y a Julian Rotter quienes en sus investigaciones han producido en un plano empírico para determinar cuáles son las condiciones que haría posible la confianza mutua en las relaciones interpersonales.

Por un lado, Morton Deutsh (1956), va afirmar que la confianza se construye cuando el individuo espera en una situación determinada predecir cuál sería la mejor opción que lo motive involucrarse en una relación interpersonal (Deutsh, 1956, p.266). Según el autor, si una persona tiene la expectativa que algo va a ocurrir y ese evento implica un interés de por medio, entonces la confianza se hace importante para poder lograr su objetivo. Para comprobar estos experimentos, el autor aplicará una metodología conductual para firmar que:

"In our experimental work to test our hypotheses, we have been utilizing a two person non-zero sum game, in which the gain or losses incurred by each person are a function of the choices made by one partner as well as the choices made by one's self [...] the essential psychological feature of the game is there is no possibility for rational individual behavior in unless the conditions of mutual trust exist". (Deutsh, 1956, p. 269).

A través de dichos experimentos, el autor pudo también comprobar que no sólo las motivaciones es lo que llevan a los hombres a cooperar en una relación de confianza mutua, sino que también, en la medida en que una persona se siente segura de sí misma, automáticamente la confianza incrementa y la probabilidad de comprometerse en un comportamiento de confianza con el otro se incrementará igualmente.

Por otro lado, el estudio sobre confianza ha sido igualmente tratado a partir de técnicas psicométricas. Para analizar el proble- 
ma de la confianza en las relaciones interpersonales, Julian Rotter construye una escala llamada "Interpersonal Trust Scale", escala psicométrica que tiene como objetivo medir y predecir el grado de confianza en las relaciones interpersonales. A través de esta escala, la confianza queda conceptualizada como las expectativas que tiene un individuo de que la promesa de otro individuo pueda ser confiable teniendo en cuenta del grado de conocimiento o de información que tienen las personas sobre otros. "According to Rotter, as situation become increasingly unfamiliar to individuals, the influence that their trusting dispositions have over their behavior grows. To put in other way, as people become more acquainted with specific others, their personal knowledge of those of others become primary driver of their thoughts and actions" (Bigley; Pearce, 1998, p. 411). Por lo tanto, para Julian Rotter "su teoría trata los factores cognoscitivos que median entre efectos de la situación y los reforzadores ambientales sobre la conducta. En concreto Julian Rotter afirmaba que los valores y las expectativas personales son las que al final determinen nuestro comportamiento" (Valk, 2008, p.25).

Se puede entonces decir que estos tipos de estudios operan bajo una lógica meramente empirista en la cual, "las hipótesis legaliformes no se refieren más que al plexo de estímulos y reacciones comportamentales" (Habermas, 1988). Como lo menciona Barbara Mitzal (1998) en estos tipos de estudios psicosociales, los trabajos sobre la confianza quedan estrechamente relacionados con una mentalidad cooperativa en la cual la mutua confianza existe a partir de las cualidades individuales de las personas como por ejemplo a partir la honestidad, la lealtad, la sinceridad, la esperanza o el altruismo.

\section{Acercamientos "holista" al estudio de la confianza:}

A diferencia de las investigaciones que se han realizado sobre el comportamiento humano en las relaciones interpersonales, se encuentran igualmente las investigaciones científicas con una visión más omnibarcadora para conceptualizar a la confianza. Como di- 
ría David Lewis (1985), la confianza no es analizada a partir de las reacciones de los hombres tienen frente al comportamiento de los demás, sino más bien a partir de las orientaciones recíprocas que son compartidas, objetivadas y simbolizadas hacia la intensión de la acción. Acá la principal crítica que se le hace a los estudios conductistas es que sus teorías sólo se centran en el comportamiento de respuestas-estímulos, sin ni siquiera preguntarse por la comprensión de las normas sociales que anteceden a la elaboración de los datos (Habermas, 1988). Por ello, la confianza como objeto de estudio, no sólo puede ser analizado en un plano meramente empírico analítico, sino que resulta necesario antes que nada, identificar en el mundo social las orientaciones recíprocas que son compartidas, objetivadas y simbolizadas hacia la intensión de la acción.

La corriente filosófica que ha contribuido y que se ha fuertemente instalado en las ciencias sociales tiene que ver por un lado con el modelo kantiano. Kant se va preguntar no sólo por cuáles son las condiciones de posibilidad del conocimiento humano sino también por cuáles son los principios prácticos que hace que los hombres deban actuar de una determinada manera. Para Kant, todas aquellas reglas que nos mandan a hacer esto o lo otro en vistas de algún fin que se suponen deseables no pueden ser considerados como leyes morales ya que siendo fruto de la experiencia, no encierran necesidad ni universalidad alguna (Bredlow, 2010). Más bien, la universalidad y la necesidad están en los principios prácticos como condición de posibilidad para que los hombres puedan actuar por su voluntad propia y como individuos autónomos:

"Principios prácticos son proposiciones que contienen una determinación universal de la voluntad que tiene varias reglas prácticas. Son subjetivos cuando la condición es considerada por el sujeto como válida solamente para su voluntad; objetivos o leyes prácticas cuando la condición se reconoce como objetiva, esto es, válida para la voluntad de todo ser racional." (Kant, 2007, p. 29). 
Haciendo de los valores algo universal y necesario para todos los hombres que hace uso de su razón, obliga a los miembros de la comunidad moral a respetarse mutuamente ya que las acciones quedan condicionadas a la ley moral. Siguiendo a Adam Seligman (1997) para Kant, el deber perfecto es lo que une a los miembros de una comunidad moral y es lo que produce el mutuo respeto entre los miembros de esta. Por lo tanto, la confianza para Kant no se encontraría en los sentimientos o en las cualidades de los comportamientos humanos, sino más bien en los valores absolutos que obligan y orientan a los hombres a través de su propia razón a respetar y a mantener sus promesas en una comunidad ideal para proteger la dignidad de los miembros de la humanidad:

"la sustentación de la dignidad de cada uno de los miembros de la humanidad constituye el aporte más especifico de la reflexión kantiana. El respeto que yo tengo por otro o que otro puede exigir de mí es el reconocimiento de la dignidad en los demás hombres [...] la humanidad es en sí misma una dignidad porque el hombre no puede ser tratado por nadie como un mero medio, sino que debe ser tratado siempre $y$ al mismo tiempo como un fin." (Papachini, 2003, p. 232).

Sólo así se hace posible mantener una armonía ideal ya que los hombres están en su obligación de obedecer a una ley moral para respetar la dignidad de los hombres, "a diferencia de los demás seres vivientes que obedecen pasivamente a las leyes del instinto." (Papachini, 2003, p. 231).

Por otro lado, Hegel también será importante para caracterizar esta visión holista de la Sociedad. Para Hegel, existen estructuras supra-personales y ominabarcadoras del ser, ancladas en la totalidad social, a diferencia de Kant, quien sostiene que una persona no debe subordinarse a ninguna otra ley distinta a la que puede darse así misma, bien sea sola o junto con otras personas (Horkheimer, 1938). La moralidad no es entonces producto de un individuo autónomo 
como en Kant, sino que más bien está situada en la estructura objetiva de las normas de la comunidad (Smith, 1989, p.72). Ya no es el individuo autónomo sino que más bien la filosofía debe centrarse en el entendimiento objetivo de aquellas estructuras totalizadoras que se incorporan en lo particular:

"Pero el Espíritu es en sí objetivo y real no sólo en cuanto tal necesidad y como reino del fenómeno; sino como idealidad del mismo, como su interioridad. Así, esta universalidad sustancial es objeto y fin para sí misma y aquella necesidad se da igualmente en la norma de la libertad." (Hegel, 1968, p. 218).

Sobre la base que acabamos de mencionar las operaciones científicas principalmente en el campo de la sociología concentraría su atención en el aspecto normativo, como condición necesaria para poder producir confianza social. Bajo esta fundamentación, "la descripción sociológica de la sociedad tiene pues grabada en símisma la primacía de la dimensión social, en la formación de lo social como una colectivos de actores individuales [...] se revela lo social como un objeto estructurado fundamentalmente de manera normati$v a$ " (Nassehi, 2011, p. 22). Lo que prevalece en este tipo de estudios tiene que ver con la creencia de que la vida social depende de las relaciones que se establecen entre los miembros de los grupos sociales y las instituciones sociales, y en la cual los actores en la sociedad deben conformarse con los valores y las normas que producen las instituciones para que puedan actuar en colectividad.

Esta visión normativa la encontramos en Habermas, quien trata de producir una programa científico reconstructivo que tiene como propósito, "dar cuenta del conocimiento pre-teórico y el dominio intuitivo de los sistemas normativos que se encuentran en la base de la producción y enjuiciamiento de manifestaciones y producciones simbólicas" (Habermas, 1988). Las operaciones científicas en el campo de las ciencias sociales concentrarían su atención en el aspecto normativo que antecede al objeto observado y esto es posible con 
la ayuda de la hermenéutica como metodología filosófica. Sólo así es posible que las Ciencias Sociales puedan comprender, más allá del dato, el mundo social de la vida sobre la cual en su objeto de estudio está enmarcado.

Resulta posible en otras palabras conocer las pretensiones de validez que tienen los sujetos en una comunidad lingüística, sin caer bajo el dominio de la razón instrumental (Horkheimer, Marcuse, Adorno) sino más bien manteniendo un interés práctico en lo que Habermas (1990) denomina el mundo de la vida, es decir, "núcleo institucional que comprende, las relaciones no gubernamentales y no económicas, así como las asociaciones de voluntarios que afianzan las estructuras de la comunicación de la esfera pública" (Habermas, 1990, p. 560). El mundo de la vida sería entonces aquella esfera en la que los hombres pueden mantener un entendimiento racional para complementar a aquellos sistemas funcionalmente racionalizados como la economía o el aparato burocrático que buscarían subordinar al interés práctico. La confianza estaría entonces en las relaciones sociales, en la comunicación racional que se dan entre los diferentes actores o asociaciones que conforman al mundo de la vida. Una esfera que se orienta como dice Barbara Mitzal (1996), a partir de valores como la justicia y la solidaridad para enfrentar al poder coercitivo del Estado y a la esfera económica que produce para el mercado.

A alero de la teoría de la sociedad producida por Habermas se despliegan investigaciones en el campo de la sociedad civil. Andrew Arato y Jean L. Cohen (2001), además de otros intelectuales como Adam Seligman (1997) y Alain Touraine (1997), serán también unos de los principales investigadores en "revitalizar" a la sociedad civil ya que lo que se busca es que "the demands of individuality can be reconciled with those of community leads to the revitalizations of the idea of civil society at the centre of which trust and mutual obligations are placed" (Mitzal, 1996, p. 2). Lo que se busca es ordenar a una sociedad compleja a partir de una base normativa que les permita a los diferentes actores integrarse en la sociedad, participar 
racionalmente en la comunicación, alcanzar un consenso o un entendimiento intersubjetivo (Habermas, 1990) y resolver sus problemas sociales y la confianza viene a ser el prerrequisito para ello.

Relacionado con la semántica de la sociedad civil, el concepto de la confianza también resulta relevante para los estudio sobre capital social. Este último hace referencia en cómo en una comunidad los miembros comparten normas y valores, colaboran, cooperan y generan redes sociales para lograr sus intereses y mantener el beneficio mutuo. Si bien el Capital Social mantiene una visión economicista que se podría caracterizar como atomicista, también se centra en cómo es posible la cooperación entre los miembros de una comunidad (actores racionales) a través de normas y valores y acá la confianza viene a ser un componente fundamental para que ellos puedan lograr sus intereses.

Fukuyama (1997) por ejemplo, entiende a la confianza como "the expectations that arises within a community of regular, honest and cooperative behavior, based on commonly shared norms, on the parts of the members of other members of that community" (Fukuyama apud Seligman, 1997, p. 77). Los miembros de la comunidad cooperan y comparten normas y valores, y la confianza está en "the art of association and its role in the creation on the economic prosperity" (Fukuyama apud Seligman, 1997, p. 77). Desde la perspectiva de la teoría de acción racional, James Coleman (1990), define a la confianza en el capital social como las expectativas que configuran la actividad económica. "Trust is nothing more or less than the considerations a rational actor applies in deciding to place a bet" (Coleman apud Rouscio, 1999, p.644). En cambio, para Robert Putmam (1996), "trust is an essential component of social capital because it lubricates cooperation (...) trust has two sources norms of reciprocity and civic engagement, and the first is likely to be a funtion of the second" (Putmam apud Levi, 1996, p. 47). La confianza es un componente del capital social que se generaliza a partir de un compromiso entre los miembros de la comunidad, orientados por 
normas sociales que obliguen a los actores a cooperar para lograr sus intereses.

Desde una visión holista de la sociedad, las operaciones científicas en el campo de las ciencias sociales concentrarían su atención en el aspecto normativo como condición necesaria para poder producir descripciones empíricas sobre cómo deben relacionarse las personas en una comunidad y por lo tanto la producción de conocimiento coloca una certeza apriorística de lo cultural, lo cual sería ella lo que produciría confianza social (Mascareño, 2009).

\section{Acercamiento sistémico al Estudio de la Confianza: Niklas Luhmann}

Una tercera alternativa al estudio de la confianza tiene que ver con una perspectiva funcional y sistémica. Para la teoría de sistemas sociales de Niklas Luhmann, la confianza viene a ser un mecanismo de reducción de complejidad, un concepto funcional que soluciona problemas en las decisiones riesgosas que enfrentamos en la sociedad, en la medida en que actuamos en la sociedad. Cabe por lo tanto detenernos y profundizar en varios aspectos sobre la teoría de los sistemas sociales del autor para poder ver el modo en que queda conceptualizado la confianza y cómo este opera en una sociedad compleja.

\section{El funcionalismo:}

El funcionalismo desde la teoría de los sistemas sociales de Niklas Luhmann, se preocupa por los problemas y sus soluciones. Desde una perspectiva funcional, las observaciones que se realiza para estudiar los fenómenos sociales no son tratados bajo una regla causal, ya que el análisis funcional, no consiste en "comprobar unidades de referencias como efectos legitimantes provocados por determinadas causas" (Luhmann 1973, p.31). No consiste en tomar "las relaciones de determinadas causas y determinados efectos, sino 
en evidenciar la confrontación que tales uniones permiten: entre causas diversas con el mismo efecto o entre efectos diversos con la misma causa" (Luhmann, 2006, p.116). El funcionalismo por lo tanto engloba en su interior a las relaciones causales agregándole un componente de indeterminación y variabilidad en las causas y en los efectos.

En la teoría de los sistemas luhmaniano, la estabilidad y la conservación de los sistemas y de su identidad son variables y contingentes. Es decir, la existencia del sistema no se debe a su estabilidad sino más bien "porque son capaces de asegurar el paso de una operación a la otra. La estabilidad misma del sistema debe entonces ser considerada como estabilidad dinámica ya que la continuidad del sistema asegura la discontinuidad de la operación" (Corsi, 1996, p.191). En este sentido, como lo plantea Niklas Luhmann, "el método funcionalista debe fundamentar precisamente la posibilidad de que algo puede ser y también no ser, de que algo es reemplazable." (Luhmann, 1973, p. 23).

El método funcionalista no es un método deductivo, "no es ningún efecto a producir, sino un esquema lógico regulador, que organiza un ámbito de comparación de efectos equivalentes" (Luhmann 1973, p. 20). Los equivalentes son funcionales y no están ajustados a la invariabilidad y a la exclusión de la indeterminación. En este sentido, "varias posibilidades de la realización aparecen como equivalentes en sentido funcional [...] se remplaza así el funcionalismo causal por un funcionalismo de las equivalencias funcionales" (Luhmann, 1973, p. 27). Como dice Knorr Cetina (2005), sin indeterminación no habría nuevas constelaciones de selecciones. Estas selecciones que dependen de los mismos cambios observados de los datos que se utilizan para construir conocimiento. Por lo que se reducirían las condiciones de posibilidad de abordar u observar los cambios que se generan en los fenómenos sociales que estamos estudiando. 


\section{Complejidad:}

Para poder profundizar sobre el concepto de la confianza desde la perspectiva de la teoría de los sistemas sociales de Niklas Luhmann, necesitamos también dejar en claro que dicho concepto opera en la complejidad misma de la sociedad moderna. Niklas Luhmann (1998) señala que la complejidad tiene que ver con la coacción de seleccionar (actualidad), lo cual significa contingencia (posibilidad) y contingencia significa riesgo, por lo que la confianza desde una perspectiva funcional "está abierto a todo tipo de posibilidades desde diferentes posiciones particulares (Luhmann, 1996, p.7) que se actualizan a cada instante, por lo que nos vemos forzados a decidir con el riesgo que ello implica.

Sosteniéndose sobre la base que la sociedad moderna es compleja, es que resulta posible observar a la confianza con una dimensión reflexiva, multidimensional y asimétrica, que no depende de una certeza apriorística como la justicia o la solidaridad. No se sostiene sobre la base de un fundamento ontológico que le permita al sujeto "integrarse en la sociedad" a partir de valores como los mencionados anteriormente ni tampoco se busca "predecir comportamientos" a partir de técnicas conductistas para determinar las características individuales que tienen los sujetos en la relaciones personales. Más bien, como bien lo menciona Bernad Barber, el estudio sobre la confianza desde una perspectiva funcional y sistémico tiene la función de otorgarnos un mapeo de expectativas cognitivas e institucionales para que los actores puedan actuar en el sistema en el cual están interactuando (Bernad Barber) para solucionar sus problemas específico de riesgo.

\section{La confianza en lo temporal, en lo objetual y en lo social}

Lo mencionado anteriormente nos lleva a plantear que la confianza implica una relación problemática con el tiempo (Luhmann, 1996). El tiempo en la sociedad moderna supondría "una ruptura de 
los horizontes de expectativas, una continua problematización de la acción futura [...] todo lo simultáneamente al instante es el motor que nos тиеve" (Beriain, 2008, p. 56). En los instantes, vivenciamos los acontecimientos, por lo que nos vemos forzados a tomar decisiones riesgosas para resolver nuestros problemas o reducir la complejidad en las relaciones sociales, en el presente. "El presente pues adquiere un estatus especial gracias a su función de integrar el tiempo y la realidad y representar un conjunto de constricciones cara a la integración del futuro y el pasado" (Luhmann, 1992, p. 170). En este sentido, uno integra expectativas (futuro) o experiencias (pasado) en el presente.

Desde la teoría de los Sistemas Sociales, resulta también necesario dejar en claro que la confianza se diferencia de aquello que mantenemos como memoria de las experiencias que vivimos al día. En otras palabras, la familiaridad a diferencia de la confianza que se orienta a un futuro, tiene que ver con una función simbólica que se orienta al pasado. En la familiaridad, "el pasado prevalece sobre el presente y el futuro [...] los elementos esenciales de la experiencia deben representarse en la historia ya que la historia es la forma más importante de reducir complejidad" (Luhmann, 1996, p. 33). En este sentido, lo familiar tiene que ver con lo vivenciado, el cual se deposita en la historia como algo que prevalece en el futuro. Esta es una precondición para la confianza o la desconfianza ya son nuestras experiencias (lo familiar) las que nos permiten operar en el mundo que conocemos (Luhmann, 1996). "We can life within a familiar world because we can [...] we never have to leave the familiar world" (Luhmann, 2000, p.2). El mundo familiar es lo que vivenciamos lo que co-experimentamos con mi otro yo. No podemos separarnos de ella, todos estamos integrados en el mundo que se nos hace socialmente comprensible (Luhmann, 1996), mientras que lo no familiar sería "lo extraño, lo sobrenatural, algo que debe ser combatido o tratado como misterioso" (Luhmann, 1996, p. 31).

Lo familiar sería un equivalente funcional a la confianza, una alternativa funcional que también reduce complejidad, con la única 
excepción que hace referencia al pasado, a lo vivido y que nos orienta a tomar decisiones para el futuro. Según Luhmann (2000), en la sociedad moderna el riesgo viene a formar parte de lo familiar ya que sabemos que en un futuro lleno de posibilidades corremos la posibilidad de sufrir decepciones. "Hace posible abrigar expectativas relativamente confiables y como consecuencia, hace posible contener los elementos restantes del riesgo" (Luhmann, 1996, p. 32). Así, "la historia deja de ser un recuerdo de las cosas experimentadas y se vuelve simplemente una estructura predeterminada que es la base para la confianza en los sistemas sociales" (Luhmann, 1996, p.34), es decir, una estructura (presente en el futuro), una cadena de acontecimientos que ordenan las acciones (Luhmann, 1998) para abrirnos nuevas perspectiva de futuro.

Además de lo temporal, el concepto de la confianza mantiene una relación con la dimensión social en el sentido que es "todo aquello que se supone lo respectivamente igual, como un alter-ego, y articula la relevancia de esta suposición para cada experiencia del mundo y fijación del sentido [...] tiene relevancia universal del mundo ya que si existe un alter ego, este es igual que ego, relevante para todos los objetos y los temas" (Luhmann, 1998, p.94). Tal como lo señalamos anteriormente, en el mundo, uno co-experimenta a cualquier persona de la misma manera formal, como otro yo (Luhmann, 1996), en un mundo que se nos hace socialmente comprensible. Todos los objetos y los temas son relevantes tanto para alter como para ego y por lo tanto, cada uno tiene un horizonte de posibilidades, un potencial para aceptar una selección riesgosa y negar otras posibilidades.

En este sentido, la contingencia será como dice Niklas Luhmann (1990), el atributo principal de la sociedad moderna para decidir no sólo frente a las posibilidades que se necesita seleccionar, sino también en cuanto a las probabilidades que se puedan cumplir en las decisiones tomadas:

"Un hecho es contingente cuando se le considera como una selección de entre otras posibilidades que, en algún sen- 
tido, siguen siendo posibilidades a pesar de la selección. Al implicar una potencia para la negación y la visibilidad de otras posibilidades, el concepto sólo puede aplicarse al sentido de la experiencia y de la acción subjetivas" (Luhmann, 1998, p.16).

En mundo socialmente comprensible, la contingencia es subjetiva y universal a la vez (Luhmann, 1998). Cada ego cuenta con un horizonte de posibilidades y debe relacionarse con la otra parte, quién también cuenta con la misma posibilidad, para seleccionar, con la posibilidad de negar opciones, y decidir qué puede ser más viable para reducir complejidad.

Estaríamos hablando de una doble contingencia, de una duplicación de alternativas en ambas partes. Talcott Parsons (1974) soluciona el problema de la doble contingencia a partir de su esquema medio/fines. Como sostiene Niklas Luhmann (2007) una acción se lleva a cabo cuando ya está establecida una diferencia entre medios y fines, es decir, cuando ya existe una concatenación de valores colectivos, que se hacen presentes en el momento en que el actor está decidido actuar. "Por ende, una colectividad funciona generalmente bajo el control de un gran número de normas particulares [...] Para institucionalizarse de una manera estable, las colectividades y los papeles deben regirse por normas y valores especificos" (Parsons, 1974, p.17). Las instituciones reproducirían normas y valores que permite que los individuos puedan alcanzar sus fines. La interacción social quedaría controlada por las normas y valores de la comunidad societal (Javala, 2003).

Sin embargo, desde la perspectiva de la teoría de los sistemas sociales de Niklas Luhmann la duplicación de la contingencia es vista como "las posibilidades de negación que pueden retenerse y estabilizarse como posibilidades reciprocamente no actualizadas sino que implicadas. La duplicación de las contingencias es posible porque este potencial se localiza en los sujetos y los sujetos pueden experimentar otros sujetos" (Luhmann, 1998, p. 17). Como lo seña- 
la Luhmann, esta duplicación comprende toda la estructura ya que existe el potencial generalizado para concebir hechos como selecciones que implican negaciones, para negar estas negaciones y para reconstruir otras posibilidades. Una selección implica negar otras posibilidades y por lo tanto, la negación es una potencia contenida en cualquier relación social.

Relacionando el problema de la dimensión social, la confianza viene a ser un prerrequisito para poder aceptar o rechazar el riesgo de que la otra parte cumpla con mis expectativas. Tanto "yo" como mi otro "yo" necesita solucionar un problema, en un presente, en una situación determinada, por lo que la confianza probabiliza una solución -con la posibilidad de negar una opción- que implica un riesgo en las relaciones sociales a las que nos enfrentamos para reducir complejidad:

"Primero que nada tiene que haber algún motivo para exponer la confianza. Tiene que haber alguna situación definida en que la persona que confía dependa de su socio; de otra manera el problema no se origina. Su comportamiento debe, entonces, comprometerlo con esta situación y hacerle correr el riesgo de que traicionen su confianza. En otras palabras debe invertir en lo que llamamos antes inversión riesgosa (Luhmann, 1996, p. 73).

La confianza implica por lo tanto, tolerar la incertidumbre de que ese futuro, la aceptación del riesgo, se haga presente sabiendo que -si el éxito no llegó- tuvo la oportunidad de seleccionar otras posibilidades. Es una inversión riesgosa, y por lo tanto, es lo que permite en un futuro negar otras posibilidades ya que si emerge una decepción, ésta se deposita en la historia como experiencia. 


\section{La confianza personal:}

Para que podamos vivenciar el mundo que se nos hace socialmente comprensible y podamos tomar decisiones riesgosas en cualquier momento del tiempo, el sistema de conciencia resulta de ser también de suma importancia. Son ellos los que nos permiten movernos, desde un cálculo interno, desde cualquier punto, hacia otras posibilidades del mundo (Luhmann, 1998). En este sentido, los sistemas de conciencia o los sistemas psíquicos son esenciales en la constitución de la sociedad, ya que con ellos podemos sondear la contingencia (Luhmann, 2006) y tener la posibilidad de realizar cálculos internos de las condiciones externas que generan riesgos (Luhmann, 2000). Desde la perspectiva de la teoría de los sistemas sociales:

"La conciencia en cuanto sistema cerrado es inaccesible para los otros sistemas autopoiéticos: ni el cuerpo ni la comunicación son capaces de determinar el flujo de pensamientos, sino que son capaces de ofrecer algunos estímulos que la conciencia es libre de elaborar en las propias formas y según sus propias estructuras" (Luhmann, 2006, p. 206).

Para la teoría luhmaniana, ni los sistemas sociales (comunicación) ni los sistemas biológicos son capaces de intervenir a la conciencia; únicamente estimulan o motivan. Por lo tanto, la socialización, "configura, pero no controla" (Jalava, 2003, p.184). En el estructuralismo funcional de Talcott Parsons, se trató de resolver el problema de la socialización a partir de un conjunto de valores colectivos, un contexto de condiciones de la acción que debía quedar presupuesto para que pudiera efectuarse la acción social (Luhmann, 2007). Como sostiene Janne Jalava (2003), para Parsons, las normas y los valores producido por la comunidad societal eran capaces de controlar la interacción y la comunicación entre los seres humanos. Esto implicaría que nuestra libertad de acción, nuestros cálculos internos que emergen de un proceso de aprendizaje estaría determina- 
da a priori por una integración normativa de las expectativas de los actores (Luhmann, 1998).

En cambio, para el paradigma de la teoría de los sistemas sociales, la socialización sería más bien un tipo de auto-socialización que configura al sistema psíquico (Vanderstraeten, 2000; Jalava, 2003). Tiene que ver, como menciona Raf Vanderstraeten (2000), con la posibilidad de leer el comportamiento de los otros a partir de una información seleccionada desde el sistema psíquico, estimulado en un contexto social (Vanderstraeten, 2000). Por lo tanto, "el proceso de aprendizaje está mediado por las experiencias del que aprende consigo mismo y es controlado por la identidad del desarrollo propio (también aprendido) del que aprende" (Luhmann, 1996, p.48). En este nivel, la confianza personal sería un prerrequisito básico para poder actuar ya que el aprendizaje (auto-aprendizaje) me permite tomar decisiones a partir de los estímulos que emergen en los contextos sociales:

"Es una precondición necesaria que la situación permita pasos selectivos, esto es, selecciones de comportamientos [...] la confianza se funda en la motivación atribuida a la conducta. La conducta en la experiencia de la cual depende la confianza debe aparecer como una expresión y reafirmación de la personalidad" (Luhmann, 1996, p. 69).

La confianza personal sería así, un elemento del sistema psíquico (Javala, 2003) que no está determinada ni institucionalmente, ni históricamente (Luhmann, 2000), "por lo que el primer prerrequisito básico para lograr la confianza personal es que las acciones humanas sean en general percibidas como determinadas personalmente" (Luhmann, 1996, p. 66). A diferencia de la confianza social que implica una relación con el otro (alter-ego), la confianza personal tiene que ver con "la reafirmación de la personalidad que uno es personalmente responsable, es la fuente de habilidad para aprender a confiar en los sistemas sociales" (Luhmann, 1996, p. 69). Y este 
"aprender a confiar" como mencionamos anteriormente, emerge de la auto-socialización que configura al sistema psíquico (Jalava 2003) para poder calcular internamente y tomar decisiones riesgosas en la sociedad.

\section{La confianza sistémica:}

Así, confiar en las otras personas o en los demás sujetos individuales, ya no se concibe automáticamente como algo obvio (Luhmann, 1996) y "como no se puede confiar en la gente, se confía en las instituciones como mediadores y generalizadores de la confian$z a$ " (Offe, 1999, p. 73). Uno tiene que aprender a tolerar las diferentes formas de considerar el mundo (Luhmann, 1998) y por lo tanto, uno tiene que aprender a confiar en los distintos sistemas funcionales que operan en la sociedad moderna.

Lo interesante de la confianza sistémica reside precisamente formación de los sistemas sociales que emergen precisamente para la coordinación de expectativas que se generan en la participación de los distintos sistemas funcionales. Por lo tanto, las normas y valores sólo son posibles si los sistemas sociales cumplen la función de resolver los problemas en la sociedad. La conformidad como dice Claus Offe (1999), será contingente sobre la probabilidad que todo el mundo sea similarmente motivado a comprometerse con sistemas sociales. Las reglas institucionales nunca pueden proveer a todas las contingencias y emergencias, éstas no son estáticas o eternas, sino contingentes e incompletas (Offe, 1999).

En tal sentido, para que haya confianza sistémica, los sistemas sociales deben tener la capacidad de reducir la complejidad y regular la incertidumbre de la sociedad. Si los sistemas sociales ofrecen confianza es porque solucionan un problema específico de riesgo y procesan las decepciones emergentes en la sociedad. De esta manera, pueden transformar las condiciones de emergencia en condiciones de persistencia (Luhmann, 1996). Es decir, tienen la posibilidad de que el presente dure para futuras acciones y abra nuevas 
perspectivas de futuro para que los individuos se comprometan con las normas y los valores:

"El autocompromiso con normas y valores es un aspecto intrínseco en la vida social. Pero este compromiso no se produce porque los hombres estimen la vida en el orden social y la honren con una especie de conceso constitucional. No existe tal contrato social, porque no existe la situación electiva presupuesta en el argumento. Pero existe [...] la necesidad de precisar tales generalizaciones donde se vuelvan riesgosas y susceptibles a la decepción. Sólo en este lugar -teóricamente subordinado y ya no fundamental - encaja la función de la normatización. Se desarrollan normas en la medida en que se requieren generalizaciones que valgan ser afirmadas contrafácticamente (contra los hechos)" (Luhmann, 1998, p. 297).

Las experiencias se generalizan en la medida en que los sistemas sociales cumplen con las expectativas de los individuos en la sociedad. Si son eficaces para hacerse cargo de las decepciones, si se generalizan estas experiencias, se pueden estabilizar y confiar en las normas ya que los sujetos individuales saben que tienen dicho recurso para poder aplicarlo en caso de que a alguien le traicionen su confianza.

El procesamiento de las decepciones y las soluciones de riesgos permitiría que, como dice Bernard Barber, se generen expectativas de persistencia (Barber) de las normas y valores que se forman para el mantenimiento del orden social. Permitía por lo tanto, un tipo de "libertad institucionalizada, es decir, una libertad coartada $y$ moderada por el orden social; la libertad como un complejo de acciones o aspecto de acciones por los cuales uno es personalmente responsable" (Luhmann, 1996, p. 69). De esta forma, se acepta que los sujetos individuales acepten los roles en sus interacciones socia- 
les y se comprometan con las obligaciones y responsabilidades que deben cumplir al momento de participar en un sistema social.

"No sólo debe participar allí lo que él es, sino que en aquello que da a conocer sobre sí mismo pone, al mismo tiempo, de relieve que él cumplirá las exigencias de los contactos comunicativos, que tiene el interés en las interacciones, que se someterá a sus normas y-lo más importante - que como individuo se presentará siendo consecuente, calculable, confiable: se mantendrá consistentemente en su individualidad" (Luhmann, 2010, p.149).

En la combinación del "yo" como individuo y en la formación de los sistemas sociales o en la confianza personal y en la confianza sistémica, es donde se refleja la posibilidad de garantizar un orden social.

\section{Conclusión:}

La producción de conocimiento que emerge en el plano en las Ciencias Sociales desde un enfoque atomicista de la sociedad busca solucionar el problema de la confianza enfocándose únicamente en las características individuales de las personas. Los estudios tratarán el problema de la confianza a partir de experimentos o técnicas psicosociales para intentar predecir las expectativas individuales en una relación interpersonal. Podemos decir que esta cultura científica nos hace creer que pisamos un terreno firme y objetivo por el hecho de saber qué es lo que medimos (Nowotny, 1992), por el hecho de tener la certeza de que las técnicas psicosociales o las metodologías conductuales permiten darle una respuesta a cómo es posible la cooperación en las relaciones interpersonales.

En un plano más macro, muchas de las descripciones o de las teorías que se producen en el sistema científico giran en torno a cuáles son las condiciones de posibilidad de mantener un orden moral a 
través una pretensión normativa en la que los valores como la justicia o la solidaridad serían las que generarían las condiciones para producir confianza social. En otras palabras, las investigaciones científicas principalmente en el campo de la sociología, centrarían en atención en cómo es posible "fomentar una conducta normativamente correcta" (Luhmann, 1996, p. 50) y sólo así sería posible la confianza.

Descrito ambos enfoques, partimos del hecho que los estudios sobre la confianza no pueden ser entendida únicamente en un plano cognitivo (comportamientos humano, relaciones interacciónales, etc), ni tampoco únicamente en un plano generalizado (normas y valores) ya que esta dos visiones quedan mutuamente incluidas cuando uno participa en un sistema social. Las expectativas individuales dependen de lo social y lo social igualmente depende de las expectativas cognitivas que cada uno de nosotros vamos construyendo en la medida en que se establecen las condiciones para participar en la comunicación de la sociedad.

En este sentido, la complejidad creciente de la sociedad moderna hace que "para generar sistemas, se requieren de más confianza personal como prerrequisito de participación, y más confianza generalizada como una condición de mejores utilizaciones de oportunidades" (Luhmann, 2000, p. 97). La confianza personal y social (o generalizada) son un pre-requisito para la participación en los sistemas funcionales y por lo tanto para la formación de éstos. De esta manera, los sistemas ofrecen recursos para solución de algún problema específico de riesgo teniendo así uno la posibilidad de aceptar o de negar una oferta comunicativa a través de nuestra capacidad reflexiva.

Cabe también aclarar que ningún sistema social puede ser el centro ni tampoco puede determinar a los demás sistemas. Más bien, "in modern society, not only everything is possible; it is even possible in different ways simultaneously. Problem generally could be handled politically, economically, judicially, educationally and of course scientifically; and all at the same time" (Schirmer; Hadamek, 2007, p. 139). Los distintos sistemas funcionales como la economía, 
el derecho, la ciencia o la misma política operan en la contingencia y en la simultaneidad. En palabras de Niklas Luhmann (1998), cada sistema tiene un tiempo propio con el todo y debe caber en el tiempo del mundo. "Ningún sistema puede avanzar más rápido que otros en el futuro y perder de esta manera la simultaneidad indispensable para los contactos con el entorno" (Luhmann, 1998, p.179). Por lo tanto y siguiendo el argumento de Danilo Zolo (2007), las ciencias sociales y políticas se encuentran actualmente en dificultades específicas precisamente porque buscan solucionar el problema del orden social, sin tomar en cuenta el alto grado de impredictibilidad de los comportamientos individuales, la complejidad creciente de las relaciones sociales y el carácter no lineal pero reflexivo de los nexos funcionales (Zolo, 1992).

Observando a la confianza desde el punto de vista funcional, multidimensional y reflexivo es que resulta abordar el problema desde una mirada de la complejidad. Concepto que opera dependiendo del sistema al cual estamos participando en la contingencia y en la simultaneidad, en el cual debemos aceptar el riesgo de que como persona el otro cumpla con mis expectativas.

Por último, esta investigación es un aporte a la producción de conocimiento sobre la confianza desde la perspectiva sistémica en América Latina. Son muy pocas, para no decir ninguna, las investigaciones que se han realizado sobre la importancia del estudio la confianza desde una perspectiva sistémica. Las investigaciones encontradas sobre la confianza, además de ser pocas, se relacionan más que todo con el capital social o con la perspectiva de la sociedad civil. Las investigaciones sobre la confianza se podría observar también en los estudios que buscan modelar el problema de la confianza para predecir las expectativas que los individuos tienen sobre otras personas, instituciones, gobiernos o valores como la democracia, justicia, libertad e igualdad. Partimos entonces del hecho de que resulta insuficiente estudiar el problema de la confianza social bajo un paradigma que predice cómo debe de comportarse la sociedad, esto reduciría las condiciones de posibilidad de observar a una so- 
ciedad compleja y por lo tanto, observar al concepto de la confianza como una dimensión reflexiva y multidimensional que no depende de una certeza apriorística.

\section{REFERENCIAS:}

ARNOLD, M.; RODRÍGUEZ, D. El perspectivismo en la teoría sociológica. Revista de Estudios Sociales. Vol. 64, nº.2, pp. 27-41, 1990.

ARATO, A.; COHEN, J. Sociedad Civil y Teoría Política. México, DF: Ed. Fondo de Cultura Económica, 2000.

BACHMANN R. Trust, Power and Control in Trans-Organizational Relations. Organizations Studies. Vol. 22, nº 2. pp. 337-365, 2001.

BARBER, B. The logic and limits of trust. New Jersey, USA: Rutgers University Press, 1983.

BERIAIN, J. Aceleración y tiranía del presente: la metamorfosis en las estructuras temporales de la modernidad. México, DF: Editorial Anthropos, 2008.

BIGLEY, G.; PEARCE, J. Straining for shared meaning in organization science: problemas of trust and distrust. Academy of Management Review, Vol. 23, nº.3, pp. 405-421, 1998.

BLAU, M. Intercambio y poder en la vida social. Barcelona: Editorial Hora, 1982.

BREDLOW, L. Kant Esencial. Madrid: Ed. Montecinos, 2010.

CETINA, K. La fábrica del conocimiento. Buenos Aires: Editorial Universidad Nacional Quilmes, 2005.

COLlingWOOD, R. La idea de la historia. México, DF: Ed. Fondo de Cultura Económica, 2004.

La idea de la naturaleza. México, DF: Ed. Fondo de Cultura Económica, 2006.

CORSI, G.; ESPOSITO E.; BARALDI C. Glosario sobre la teoría social de Niklas Luhmann. México, DF; Ed. Anthropos, 1996. 
CHOUDHURY, E. Trust in Administration: an Integrative Approach to Optimal Trust. Administration \& Society. Vol. 40, n6, pp. 586. 620, 2008.

DEUTSCH, M. Trust and Suspicion. The Journal of Conflict Resolution, Vol. 2, no.4, pp. 265-279, 1958.

DURKHEIM, E. La división social del trabajo. Barcelona: Ed Akal, 2001.

FUKUYAMA, F. La confianza: las virtudes sociales y la capacidad de generar prosperidad. Buenos Aires: Editorial Atlántida, 1996.

HABERMAS, J. La lógica de las Ciencias Sociales. Madrid: Editorial Tecnos, 1988.

. Teoría de la Acción Comunicativa. Vol. II: Crítica de la razón funcionalista. México, DF: Editorial Taurus, 1990.

HEGEL, G. W. F. Filosofía del Derecho. Buenos Aires: Ed. Claridad, 1967.

HOBBES, T. Leviathan. Tomo I. México, DF: Ed. Gernika, 1997. Elementos filosóficos del ciudadano. Buenos Aires: Ed. Hydra, 2010.

HOFFMANN A. A Conceptualization of Trust in International Relations. European Journal of International Relations. Vol. 8, n³. pp. 375401, 2002.

HUME, D. Investigación sobre la moral. Buenos Aires: Ed. Losada, 2003. - Tratado de la Naturaleza Humana. México, DF: Ed. Porrúa, 2005.

JAVALA, J. From Norms to Trust: The Luhmanian Connections between Trust and System. European Journal of Social Theory, Vol. 6, $\mathrm{n}^{\mathrm{o}} .2$, pp.173-190, 2003.

JERPHAGNON, L. (Dir.). Dictionnaire des grandes philosophies. Toulouse: Privat, 1983.

LEWIS, D; WEIGERT, A. Social Atomism, Holism and Trust. The Sociological Quartely, Vol. 26, nº. 4, pp. 455-471, 1985. 
LEVI, M. Social and Unsocial Capital: A review Essays of Robert Putmam's Making Democracy Work. Politics \& Society, Vol. 24, nº.1, pp.45$55,1996$.

LUHMANN, N. La Ilustración Sociológica y otros ensayos. Buenos Aires: Editorial Sur, 1973.

Essays on Self-Reference. Columbia: Columbia University Press, 1990.

. Confianza. México, DF: Editorial Anthropos, 1996.

. Sistemas Sociales: Lineamientos para una teoría general., DF, México, DF: Editorial Anthropos, 1998.

. Teoría de los Sistemas Sociales. México, DF: Universidad Iberoamericana, 1998.

. Familiarity, Confidence, Trust: Problems and Alternatives. In: GAMBETTA, Diego (Ed.). Trust: Making and Breaking Cooperative Relations. New York: Basil Blackwell, 2000.

La Sociedad de la Sociedad. México, DF: Editorial Herder, 2007.

Los derechos fundamentales como institución. México, DF: Universidad Iberoamericana, 2010.

MASCAREÑO, A. Contingencia, necesidad e imposibilidad en la semántica de América Latina. In: FARÍAS, I. y OSSANDON, J. (Eds.). Observando Sistemas II. Chile: Ed. UIA, 2009.

MIZTAL, B. Trust Cooperation: the democratic public sphere. Journal of Sociology, Vol. 37, no.4, pp. 371-386, 2001.

MURPHY J. Building trust in economics spheres. Progress in Human Geography. Vol. 30, n. 4, pp. 427-450, 2006.

NASSEHI, A. La teoría de la diferenciación funcional en el horizonte de sus críticas. Revista MAD, n.24, pp 1-29, 2011.

NOWOTNY, H. Estructuración y medición del tiempo: sobre la interrelación entre instrumentos de medición del tiempo y el tiempo social. In: 
RAMOS, R. (Ed.). Tiempo y Sociedad. DF. México, DF: Siglo XXI, 1992.

KANT, E. Crítica de la razón pura. Madrid: Ed. Alfaguara, 2003. Crítica de la razón práctica. Buenos Aires: Ed. Lozada, 2007.

OFFE, C. How can we trust our fellowships? In: WARREN, Mark (Ed.). Trust and Democracy. Cambridge: Cambridge University Press, 1999.

PAPACCHINI. A. Filosofía y Derechos Humanos. Cali: Universidad del Valle, 2003.

PARSONS, T. El sistema de las sociedades modernas. México, DF: Editorial Trillas, 1974.

SELIGMAN, A. The problem of trust. Princeton: Princeton University Press, 1997.

SIMMEl, G. The Philosophy of Money. New York: Routledge, 2011.

SMITH, S. Hegel Critique of Liberalism. Right in Context. Chicago: University of Chicago Press, 1989.

SCHIRMER, W.; HADAMEK, C. Steering as paradox: the ambiguous role of the Political System in Modern Society. Cybernetic and Human Knowing, Vol. 14, n. 2-3, pp. 133-150, 2007.

SZTOMPKA P. Trust in Science. Robert K. Merton's Inspirations. Journal of Classical Sociology. Vol. 7, nº. 2, pp. 211-220, 2007.

TOURAINE, A. Podremos vivir juntos: iguales y diferentes. México, DF: Fondo de Cultura Económica, 1997.

VANDERSTRAETEN, R. Autopoiesis and Socialization: On Luhmann's reconceptualization of Communication and Socialization. British Journal of Sociology, Vol. 51, nº. 3, pp. 581-598, 2000.

VANDERSTRAETEN, R. Luhmann on Socialization and Education. Educational Theory, Vol. 50, no.1, pp. 1-23, 2000.

ZOLO, D. Democracy and Complexity. Pennsylvania: The Pennsylvania State University Press, 1992. 
La tragedia de la ciencia política. Revista Universitaria de Ciencias Sociales, Vol. 11, nº.14, pp. 51-69, 2007.

\section{Online:}

HORKHEIMER, Max (enero 2012). La situación actual de la filosofía social y las tareas de un Instituto de investigación social (1931).

Disponible en: http://santiagocastrogomez.sinismos.com/ blog/?p=235

[fecha de acceso: marzo 2012]

VALK, Astrid (junio 2008). Comparación de la capacidad predictiva del modelo de los cinco factores y el modelo de las evaluaciones centrales en la satisfacción laboral. Disponible en:

http://catarina.udlap.mx/u_dl_a/tales/documentos/moor/ valckx_g_a/capitulo1.pdf

[fecha de acceso: marzo 2012]

Recebido em: 24/04/2012

Aprovado em: 29/05/2012 